\title{
Some Subordination Results for Fractional Integral Involving Wanas Differential Operator
}

\author{
Abbas Kareem Wanas \\ Department of Mathematics, College of Science, University of Al-Qadisiyah, Diwaniyah, Iraq \\ e-mail: abbas.kareem.w@qu.edu.iq
}

\begin{abstract}
In the present paper, we establish some differential subordination properties for analytic functions defined in the open unit disk associated with the fractional integral by using Wanas differential operator.
\end{abstract}

\section{Introduction}

Let $\mathcal{H}(U)$ denote the space of all analytic functions in the open unit disk $U=$ $\{z \in \mathbb{C}:|z|<1\}$. Assume that $A_{n}=\left\{f \in \mathcal{H}(U), f(z)=z+a_{n+1} z^{n+1}+\cdots, z \in U\right\}$ with $A_{1}=A$.

Given two functions $f$ and $g$ which are analytic in $U$, we say that $f$ is subordinate to $g$, written $f \prec g$ or $f(z) \prec g(z)(z \in U)$, if there exists a Schwarz function $w$ which is analytic in $U$ with $w(0)=0$ and $|w(z)|<1$ such that $f(z)=g(w(z)),(z \in U)$. In particular, if the function $g$ is univalent in $U$, then we have the following equivalent (see [5]), $f \prec g \Leftrightarrow f(0)=g(0)$ and $f(U) \subset g(U)$.

Let $\psi: \mathbb{C}^{3} \times U \rightarrow \mathbb{C}$ and let $h$ be univalent function in $U$. If $p$ is analytic in $U$ and satisfies the (second-order) differential subordination

Received: December 6, 2019; Accepted: February 12, 2020

2010 Mathematics Subject Classification: 30C45, 30A20, 34A40.

Keywords and phrases: analytic function, differential subordination, fractional integral, Wanas differential operator.

Copyright (C) 2020 Abbas Kareem Wanas. This is an open access article distributed under the Creative Commons Attribution License, which permits unrestricted use, distribution, and reproduction in any medium, provided the original work is properly cited. 


$$
\psi\left(p(z), z p^{\prime}(z), z^{2} p^{\prime \prime}(z) ; z\right) \prec h(z),
$$

then $p$ is called a solution of the differential subordination. The univalent function $q$ is called a dominant of the solutions of the differential subordination, or more simply dominant if $p \prec q$ for all $p$ satisfying (1.1).

Definition 1.1 [6]. For $f \in A$. The Wanas differential operator is defined by

$$
W_{\alpha, \beta}^{k, \delta} f(z)=z+\sum_{n=2}^{\infty}\left[\sum_{m=1}^{k}\left(\begin{array}{l}
k \\
m
\end{array}\right)(-1)^{m+1}\left(\frac{\alpha^{m}+n \beta^{m}}{\alpha^{m}+\beta^{m}}\right)\right]^{\delta} a_{n} z^{n},
$$

where $\alpha \in \mathbb{R}, \beta \geq 0$ with $\alpha+\beta>0, m, \delta \in \mathbb{N}_{0}=\mathbb{N} \bigcup\{0\}$.

Definition 1.2 [1]. The fractional integral of order $\lambda(\lambda>0)$ is defined for a function $f$ by

$$
D_{z}^{-\lambda} f(z)=\frac{1}{\Gamma(\lambda)} \int_{0}^{z} \frac{f(t)}{(z-t)^{1-\lambda}} d t
$$

where $f$ is an analytic function in a simply-connected region of the $z$-plane containing the origin, and the multiplicity of $(z-t)^{\lambda-1}$ is removed by requiring $\log (z-t)$ to be real, when $(z-t)>0$.

From Definition 1.1 and Definition 1.2, we conclude that

$$
\begin{aligned}
D_{z}^{-\lambda} W_{\alpha, \beta}^{k, \delta} f(z)= & \frac{1}{\Gamma(2+\lambda)} z^{1+\lambda} \\
& +\sum_{n=2}^{\infty} \frac{\Gamma(n+1)}{\Gamma(n+1+\lambda)}\left[\sum_{m=1}^{k}\left(\begin{array}{c}
k \\
m
\end{array}\right)(-1)^{m+1}\left(\frac{\alpha^{m}+n \beta^{m}}{\alpha^{m}+\beta^{m}}\right)\right]^{\delta} a_{n} z^{n+\lambda} .
\end{aligned}
$$

It is easily verified from (1.4) that

$$
\begin{aligned}
z\left(D_{z}^{-\lambda} W_{\alpha, \beta}^{k, \delta} f(z)\right)^{\prime}= & {\left[1+\sum_{m=1}^{k}\left(\begin{array}{c}
k \\
m
\end{array}\right)(-1)^{m+1}\left(\frac{\alpha}{\beta}\right)^{m}\right] D_{z}^{-\lambda} W_{\alpha, \beta}^{k, \delta+1} f(z) } \\
& +\left[\lambda-\sum_{m=1}^{k}\left(\begin{array}{l}
k \\
m
\end{array}\right)(-1)^{m+1}\left(\frac{\alpha}{\beta}\right)^{m}\right] D_{z}^{-\lambda} W_{\alpha, \beta}^{k, \delta} f(z) .
\end{aligned}
$$


To establish our main results, we require the following lemma.

Lemma 1.1 [5]. Let $g$ be a convex function in $U$ and let $h(z)=g(z)+n \gamma g^{\prime}(z)$, for $z \in U$, where $\gamma>0$ and $n$ is a positive integer.

$$
\begin{gathered}
\text { If } p(z)=g(0)+p_{n} z^{n}+p_{n+1} z^{n+1}+\cdots, \text { for } z \in U, \text { is analytic in } U \text { and } \\
p(z)+\gamma z p^{\prime}(z) \prec h(z),
\end{gathered}
$$

for $z \in U$, then

$$
p(z) \prec g(z)
$$

and this result is sharp.

Such type of study was carried out by various authors for another classes (see [2, 3, 4]).

\section{Main Results}

Theorem 2.1. Let $g$ be a convex function such that $g(0)=1$ and let $h$ be the function $h(z)=g(z)+z g^{\prime}(z)$, for $z \in U$. If $f \in A$ satisfies the differential subordination:

$$
\begin{aligned}
& \frac{(1-\lambda) \lambda !\left[1+\sum_{m=1}^{k}\left(\begin{array}{c}
k \\
m
\end{array}\right)(-1)^{m+1}\left(\frac{\alpha}{\beta}\right)^{m}\right]}{z^{1+\lambda}} D_{z}^{-\lambda} W_{\alpha, \beta}^{k, \delta+1} f(z) \\
& -\frac{\lambda !\left[\lambda\left(\lambda-\left[1+\sum_{m=1}^{k}\left(\begin{array}{c}
k \\
m
\end{array}\right)(-1)^{m+1}\left(\frac{\alpha}{\beta}\right)^{m}\right]\right)+\sum_{m=1}^{k}\left(\begin{array}{c}
k \\
m
\end{array}\right)(-1)^{m+1}\left(\frac{\alpha}{\beta}\right)^{m}\right]}{z^{1+\lambda}} D_{z}^{-\lambda} W_{\alpha, \beta}^{k, \delta} f(z) \\
& +\frac{\lambda !}{z^{-1+\lambda}}\left(D_{z}^{-\lambda} W_{\alpha, \beta}^{k, \delta} f(z)\right)^{\prime \prime} \prec h(z),
\end{aligned}
$$

then

$$
\frac{\lambda !\left(D_{z}^{-\lambda} W_{\alpha, \beta}^{k, \delta} f(z)\right)^{\prime}}{z^{\lambda}} \prec g(z) .
$$


Proof. Suppose that

$$
p(z)=\frac{\lambda !\left(D_{z}^{-\lambda} W_{\alpha, \beta}^{k, \delta} f(z)\right)^{\prime}}{z^{\lambda}} .
$$

Then $p$ is analytic in $U$ and $p(0)=1$.

Differentiating both sides of (2.2) with respect to $z$ and using the identity (1.5), we have

$$
\begin{aligned}
& p(z)+z p^{\prime}(z) \\
= & \frac{(1-\lambda) \lambda !\left[1+\sum_{m=1}^{k}\left(\begin{array}{c}
k \\
m
\end{array}\right)(-1)^{m+1}\left(\frac{\alpha}{\beta}\right)^{m}\right]}{z^{1+\lambda}} D_{z}^{-\lambda} W_{\alpha, \beta}^{k, \delta+1} f(z) \\
& -\frac{\lambda !\left[\lambda\left(\lambda-\left[1+\sum_{m=1}^{k}\left(\begin{array}{c}
k \\
m
\end{array}\right)(-1)^{m+1}\left(\frac{\alpha}{\beta}\right)^{m}\right]\right)+\sum_{m=1}^{k}\left(\begin{array}{c}
k \\
m
\end{array}\right)(-1)^{m+1}\left(\frac{\alpha}{\beta}\right)^{m}\right]}{\left.z^{1+\lambda}\right]} D_{z}^{-\lambda} W_{\alpha, \beta}^{k, \delta} f(z) \\
& +\frac{\lambda !}{z^{-1+\lambda}}\left(D_{z}^{-\lambda} W_{\alpha, \beta}^{k, \delta} f(z)\right)^{\prime \prime} .
\end{aligned}
$$

In view of the subordination (2.1), we find from (2.3) that

$$
p(z)+z p^{\prime}(z) \prec h(z) .
$$

Making use of Lemma 1.1, yields $p(z) \prec g(z)$. By (2.2), we obtain

$$
\frac{\lambda !\left(D_{z}^{-\lambda} W_{\alpha, \beta}^{k, \delta} f(z)\right)^{\prime}}{z^{\lambda}} \prec g(z) .
$$

This completes the proof of the theorem.

Theorem 2.2. Let $g$ be a convex function such that $g(0)=1$ and let $h$ be the function $h(z)=g(z)+z g^{\prime}(z)$, for $z \in U$. If $f \in A$ satisfies the differential subordination:

$$
\frac{1}{\left[\lambda-\sum_{m=1}^{k}\left(\begin{array}{c}
k \\
m
\end{array}\right)(-1)^{m+1}\left(\frac{\alpha}{\beta}\right)^{m}\right]}\left[\frac{\lambda(1+\lambda) !\left[1+\sum_{m=1}^{k}\left(\begin{array}{c}
k \\
m
\end{array}\right)(-1)^{m+1}\left(\frac{\alpha}{\beta}\right)^{m}\right]}{z^{1+\lambda}} D_{z}^{-\lambda} W_{\alpha, \beta}^{k, \delta+1} f(z)\right.
$$




$$
\left.-\frac{(1+\lambda) ! \sum_{m=1}^{k}\left(\begin{array}{l}
k \\
m
\end{array}\right)(-1)^{m+1}\left(\frac{\alpha}{\beta}\right)^{m}}{z^{\lambda}}\left(D_{z}^{-\lambda} W_{\alpha, \beta}^{k, \delta} f(z)\right)^{\prime}\right] \prec h(z),
$$

then

$$
\frac{(1+\lambda) ! D_{z}^{-\lambda} W_{\alpha, \beta}^{k, \delta} f(z)}{z^{1+\lambda}} \prec g(z) .
$$

Proof. Suppose that

$$
p(z)=\frac{(1+\lambda) ! D_{z}^{-\lambda} W_{\alpha, \beta}^{k, \delta} f(z)}{z^{1+\lambda}} .
$$

Then $p$ is analytic in $U$ and $p(0)=1$.

Differentiating both sides of (2.5) with respect to $z$ and the identity (1.5), we get

$$
\begin{aligned}
& p(z)+z p^{\prime}(z) \\
&=\left.\frac{1}{\left[\lambda-\sum_{m=1}^{k}\left(\begin{array}{c}
k \\
m
\end{array}\right)(-1)^{m+1}\left(\frac{\alpha}{\beta}\right)^{m}\right]}\right] \frac{\lambda(1+\lambda) !\left[1+\sum_{m=1}^{k}\left(\begin{array}{c}
k \\
m
\end{array}\right)(-1)^{m+1}\left(\frac{\alpha}{\beta}\right)^{m}\right]}{z^{1+\lambda}} D_{z}^{-\lambda} W_{\alpha, \beta}^{k, \delta+1} f(z) \\
&\left.-\frac{(1+\lambda) ! \sum_{m=1}^{k}\left(\begin{array}{c}
k \\
m
\end{array}\right)(-1)^{m+1}\left(\frac{\alpha}{\beta}\right)^{m}}{z^{\lambda}}\left(D_{z}^{-\lambda} W_{\alpha, \beta}^{k, \delta} f(z)\right)^{\prime}\right] .
\end{aligned}
$$

From the subordination (2.4) and the equation (2.6), we deduce that

$$
p(z)+z p^{\prime}(z) \prec h(z) .
$$

Making use of Lemma 1.1, yields $p(z) \prec g(z)$. By (2.5), we obtain the required result.

Theorem 2.3. Let $g$ be a convex function such that $g(0)=1$ and let $h$ be the function $h(z)=g(z)+z g^{\prime}(z)$, for $z \in U$. If $f \in A$ satisfies the differential 
subordination:

$$
\left[\frac{z\left(D_{z}^{-\lambda} W_{\alpha, \beta}^{k, \delta+1} f(z)\right)}{D_{z}^{-\lambda} W_{\alpha, \beta}^{k, \delta} f(z)}\right]^{\prime} \prec h(z),
$$

then

$$
\frac{D_{z}^{-\lambda} W_{\alpha, \beta}^{k, \delta+1} f(z)}{D_{z}^{-\lambda} W_{\alpha, \beta}^{k, \delta} f(z)} \prec g(z) .
$$

Proof. Suppose that

$$
p(z)=\frac{D_{z}^{-\lambda} W_{\alpha, \beta}^{k, \delta+1} f(z)}{D_{z}^{-\lambda} W_{\alpha, \beta}^{k, \delta} f(z)} .
$$

Note that

$$
\begin{aligned}
p(z)= & \frac{\frac{1}{\Gamma(2+\lambda)} z^{1+\lambda}+\sum_{n=2}^{\infty} \frac{\Gamma(n+1)}{\Gamma(n+1+\lambda)}\left[\sum_{m=1}^{k}\left(\begin{array}{c}
k \\
m
\end{array}\right)(-1)^{m+1}\left(\frac{\alpha^{m}+n \beta^{m}}{\alpha^{m}+\beta^{m}}\right)\right]^{\delta+1} a_{n} z^{n+\lambda}}{\frac{1}{\Gamma(2+\lambda)} z^{1+\lambda}+\sum_{n=2}^{\infty} \frac{\Gamma(n+1)}{\Gamma(n+1+\lambda)}\left[\sum_{m=1}^{k}\left(\begin{array}{c}
k \\
m
\end{array}\right)(-1)^{m+1}\left(\frac{\alpha^{m}+n \beta^{m}}{\alpha^{m}+\beta^{m}}\right)\right]^{\delta} a_{n} z^{n+\lambda}} \\
= & \frac{1}{\Gamma(2+\lambda)}+\sum_{n=2}^{\infty} \frac{\Gamma(n+1)}{\Gamma(n+1+\lambda)}\left[\sum_{m=1}^{k}\left(\begin{array}{c}
k \\
m
\end{array}\right)(-1)^{m+1}\left(\frac{\alpha^{m}+n \beta^{m}}{\alpha^{m}+\beta^{m}}\right)\right]^{\delta+1} a_{n} z^{n-1} \\
& \frac{1}{\Gamma(2+\lambda)}+\sum_{n=2}^{\infty} \frac{\Gamma(n+1)}{\Gamma(n+1+\lambda)}\left[\sum_{m=1}^{k}\left(\begin{array}{c}
k \\
m
\end{array}\right)(-1)^{m+1}\left(\frac{\alpha^{m}+n \beta^{m}}{\alpha^{m}+\beta^{m}}\right)\right]^{\delta} a_{n} z^{n-1}
\end{aligned}
$$

Then $p$ is analytic in $U$ and $p(0)=1$. A simple computation using (2.8) gives

$$
\begin{aligned}
& {\left[\frac{z\left(D_{z}^{-\lambda} W_{\alpha, \beta}^{k, \delta+1} f(z)\right)}{D_{z}^{-\lambda} W_{\alpha, \beta}^{k, \delta} f(z)}\right]^{\prime} } \\
= & \frac{D_{z}^{-\lambda} W_{\alpha, \beta}^{k, \delta+1} f(z)}{D_{z}^{-\lambda} W_{\alpha, \beta}^{k, \delta} f(z)}
\end{aligned}
$$




$$
\begin{aligned}
& +z \frac{D_{z}^{-\lambda} W_{\alpha, \beta}^{k, \delta} f(z)\left(D_{z}^{-\lambda} W_{\alpha, \beta}^{k, \delta+1} f(z)\right)^{\prime}-D_{z}^{-\lambda} W_{\alpha, \beta}^{k, \delta+1} f(z)\left(D_{z}^{-\lambda} W_{\alpha, \beta}^{k, \delta} f(z)\right)^{\prime}}{\left(D_{z}^{-\lambda} W_{\alpha, \beta}^{k, \delta} f(z)\right)^{2}} \\
& =p(z)+z p^{\prime}(z) .
\end{aligned}
$$

In the light of (2.9), the subordination (2.7) can be written as

$$
p(z)+z p^{\prime}(z) \prec h(z) .
$$

Making use of Lemma 1.1, yields $p(z) \prec g(z)$. By (2.8), we have

$$
\frac{D_{z}^{-\lambda} H_{m}^{l}\left[\alpha_{1}+1\right] f(z)}{D_{z}^{-\lambda} H_{m}^{l}\left[\alpha_{1}\right] f(z)} \prec g(z) .
$$

which completes the proof of the theorem.

\section{References}

[1] N. E. Cho and M. K. Aouf, Some applications of fractional calculus operators to a certain subclass of analytic functions with negative coefficients, Turkish J. Math. 20 (1996), 553562.

[2] A. A. Lupaş, A note on differential subordinations using Sălăgean and Ruscheweyh operators, ROMAI J. 6(1) (2010), 1-4.

[3] A. A. Lupaş, Certain differential subordinations using a generalized Sălăgean operator and Ruscheweyh operator, Fract. Calc. Appl. Anal. 13(4) (2010), 355-360.

[4] A. A. Lupaş, Certain differential subordinations using Sălăgean and Ruscheweyh operators, Acta Univ. Apulensis Math. Inform. 29 (2012), 125-129.

[5] S. S. Miller and P. T. Mocanu, Differential Subordinations: Theory and Applications, Series on Monographs and Textbooks in Pure and Applied Mathematics, Vol. 225, Marcel Dekker Inc., New York and Basel, 2000.

[6] A. K. Wanas, New differential operator for holomorphic functions, Earthline J. Math. Sci. 2(2) (2019), 527-537. https://doi.org/10.34198/ejms.2219.527537 